\title{
Premières constatations sérologiques sur l'incidence de la maladie de Wesselsbronn et de la Fièvre de la Vallée du Rift chez les ovins et les ruminants sauvages du Tchad et du Cameroun
}

\author{
por Y. MAURICE \\ avec l'aide technique de Madame BAILLE
}

\begin{abstract}
RÉSUMÉ
L'auteur a montré, en utilisant la technique classique d'inhibition de l'hémagglutination, la présence d'anticorps conlre les virus de la maladie de Wesselsbronn et de la Fièvre de la Vallée du Rift à un laux souvent élevé, chez les petits ruminants du Tchad et du Cameroun et chez des ruminants sauvages du Tchad.

Dans certaines régions du Cameroun on constate ainsi que chez les ovins que 72 p. 100 présentent des anticorps signant l'existence de lo maladie de Wesselsbronn, tandıs que 45 p. 100 montrent des anticorps attestant la présence de la Fièvre de la Vallée du Rift.

Si ces deux maladies ont été suspeciées par certains, elles n'ont jamais été reconnues ou décrites dans ces deux pays. Ceci n'est pas pour surprendre, quand on connaît la symptomatologie très fruste de la maladie de Wesselsbronn qui passe très souvent inaperçue el l'existence dans certaınes régions d'Afrıque de formes inapparentes de Fièvre de la Vallée du Riff. L'existence de cette dernière pourraıt explıquer, chez les bovins, cerlains avortements d'étiologie jusqu'ici inconnue.

La gazelle, le darnalisque, le bubale, l'oryx, le cob et le buffle jouent vralsemblablement un rôle dans l'épızootıolcgie de celle affection.
\end{abstract}

\section{1. - LA MALADIE DE WESSELSBRONN}

ET LA FIĖVRE DE LA VALLÉE DU RIFT

10 La maladie de Wesselsbronn.

La maladie de Wesselsbronn est une maladie à virus transmise par des Arthropodes et caractérisée par de la fière et des avortements chez les ovins, ainsi que par des mortalités chez les agneaux nouveaux-nés.

L'affection ful identifiée pour la première fois en 1954-1955 dans un élevage de moutons mérinos de la région de Wesselsbronn, dans l'Etat libre d'Orange. On observa de la mortalité des agneaux nouveaux-nés pendant la premıère semaine et de l'avortement chez les brebis. Le virus fut alors isolé par WEISS, HAIG et ALEXANDER (1956) (17). Peu de temps après, deux souches du même virus furent isolées par SMITHBURN, KOKERNOT et de MEILLON (1956) dans le Nord Zululand. En 1956, toujour 5 en Afrique du Sud, on constata que la maladie de Wesseisbronn et la Fièvre de la Vallée du Rift pouvaient coexıster dans les mêmes exploitations. La distribution et l'épizootiologie de 
ces deux affections sont à peu près les mêmes. La maladie a été également reconnue dans la province du Cap, en Afrique du Sud, au Nyassaland, en Rhodésie du Nord et du Sud.

D'une façon générale, la symptomatologie esł fruste ef la maladie passe le plus souvent inaperçue surtout lorsqu'il s'agit d'élevage extensif où les animaux sont morns suivis que dans une exploitation.

On n'observe aucun symptôme chez les brebis adultes non gestantes. Les agneaux nouveauxnés peuvent montrer des signes de faiblesse et de l'inappétence. Dans quelques cas la mort survient. Les manifestations les plus caractéristıques de l'infection par le virus de Wesselsbronn sont les avortements. Chez les femelles pleines, le taux de mortalıté dans certaines régions peut atteındre 20 p. 100.

Les agneaux qui meurent de la maladie montrent une tuméfaction de la rate et des phénomènes dégénératifs au niveau du foie qui peut prendre un aspect vert-jaune et être friable. Les femelles pleines qui meurent montrent également des lésions au niveau du foie. La clinique permet difficilement de différencier la maladie de Wesselsbronn et la Fièvre de la Vallée du Rift.

\section{$2^{\circ}$ La Fièvre de la Vallée du Rift.}

La Fièvre de la Vallée du Rıft, maladie à virus transmise également par des Arthropodes et qui affecte principalement les rumınants, se caractérise par une courte période d'incubatıon, des avortements, une forte mortalité chez les angeaux, chevreaux et vecux, une allure épizootique et des lésıons hépatiques typıques.

La maladie fut observée en 1912 à Nalvasha au Kenya (STORDY) (16). Dix huit ans plus tard, DAUBNEY, HUDSON et GARNAN (5) étudiaient la seconde épızootie et identifiaient l'agent causal qu'ils appelèrent « Virus de la Fièvre de la Vallée du Rift ».

En 1948 SMITHBURN, HADDOW ef GILLET (15) isolent le virus à partir de moustiques dans la forêt de Semlikı en Ouganda. En 1950-51, la maladie faıt pour la première fois son apparition en Afrique du Sud (ALEXANDER et DICKSON) (1), MUNDEL et GEAR (10), dans l'Etat libre d'Orange, dans la province du Cap, dans la région de Kimberley. En 1955 elle est signalée dans le Sud-Ouest Africaın.
La maladie est diagnostiquée pour la première fois au Tanganyka en 1956 et au Nigéric en 1958 (6). Sans avorr isolé le virus, FINDLAY, STEFANOPOULO et MAC CALLUM (8) en 1936 montrent cependant par la sérologie, l'existence de la maladie en Ouganda, au Mali, au Soudan et au Gabon. En 1954, PELISSIER et ROUSSELOT (11) constatent que des sérums de singes du Congo présentent des anticorps fixant le complément vis-à-vis du virus de la Fièvre de la Vallée du Rift. Récemment au Kenya, SCOTT, WEDDEL et REID (14) ont pu montrer la fréquence des infections inapparentes : c'est cins! que 19 p. 100,11 p. 100 et 16 p. 100 des sérums de vache ayant avorté en 1952, 1953, 1954, présentent des anticorps contre le virus de la Fièvre de la Vallée du Rift. En 1957, SHONE, en Rhodésie du Sud met également en évidence des anticorps neutralisants dans le sérum du bétail des régions de Salisbury et de Matobo.

Dans les conditions naturelles, les agneaux nouveaux-nés sont les animaux les plus sensibles. On peut noter une mortalité de 90 p. 100 des animaux. La maladie naturelle cause la mort des agneaux dès le $3^{\mathrm{e}}$ jour. Les symptômes que I'on rencontre dans la forme aiguë sont caractérisés par une réaction fébrile, un pouls rapide, un pas chancelant, un jetage mucopurulent, des vomissements, une diarrhée hémorragique et des avortements chez les femelles pleines. Si le taux de mortalité chez les agneaux est élevé, chez les adultes il est de 20 d̀ 30 p. 100. La forme subaigue est observée chez les moutons adultes et les bovins : une réaction fébrile durant de 1 à 4 jours est accompagnée d'ınappétence et de faiblesse générale.

Les avortements surviennent durant la phase aigue ou la phase de convalescence de la maladie; environ 20 p. 100 des femelles pleines avortent. Chez les bovins, la mortalité est moins grande, moins de 10 p. 100 et dans beaucoup de cas, l'avortement est la seule trace de la maladie qu'on puisse déceler par la sérologie.

La chèvre mantre des symptômes identiques à ceux du mouton.

L'homme et le singe sont également sensibles à l'infection. DAUBNEY et HUDSON (1932) (3) ont montré que le buffle africain peut être atteint de la maladie. On pense que c'est également le cas pour de nombreux ruminants sauvages car pendant l'épidémie de 1951 en Afrique 
du Sud on rapporte que de nombreux animaux sauvages sont moris ou ont avorté dans des régions d'épizootie de Fièvre de la Vallée du Rift (GEAR et Coll.) (1951) (9), ALEXANDER et DICKSON 1951 (1).

Les lésions macroscopiques ont été décrites par DAUBNEY et FINDLAY (1931-1933). Chez l'agneau, le foie est hypertrophié et montre de petits foyers nécrotiques d'environ $1 \mathrm{~mm}$ de diamètre; l'organe est plus pâle et quelquefois complètement jaune. Chez le mouton adulte, le foie est comme tâcheté de brun rouge, aspect dù à la formation de petits foyers nécrotiques qui sont plus pâles que le tissu normal.

Chez les bovins, les lésions sont à peu près identiques à celles trouvées chez le mouton. SCHULZ (1951) (12) (13) a cependant constaté en plus une stomatite aigué avec des érosions sur les lèvres, la langue et les joues, une nécrose au nivecu de la mamelle et du scrotum, des hémorragies au niveau des régions non pigmentées de la peau et parfois de l'ascite.

La Fièvre de la Vallée du Riff et la maladie de Wesselsbronn n'ont jamais été identifiées au Tchad ni au Cameroun. Si elles ont été quelquefois soupçonnées $(\bullet$ ), aucune identification de virus ni aucune investigation sérologique n'ont jusqu'à présent été effectuées, dans ces régions. La question se posait de savoir si, étant donné la symptomatologie assez fruste de la maladie de Wesselsbronn et l'existence dans certaines régions d'Afrique de formes inapparentes de la Fièvre de la Vallée du Rift, ces deux viroses n'étaient pas en fait une composante de la pathologie des bovins et des petits ruminants au Tchad et au Cameroun.

Des sérums d'animaux sauvages ont été également analysés, pour étudier la possibilité pour ces animaux, de jouer un rôle dans l'épizootıologie de la maladie.

\section{Il. - MATÉRIEL ET TECHNIQUE}

\section{A) Matériel.}

Les réactions qui sont décrites ca-dessous ont été effectuées avec des antigènes et des sérums

(-) Le ropporl annuel du laboratoire de Farcha pour l'année 1959 (p. 12) mentionne la suspicion de Fièvre de la vallée du Rifl chez un boeuf. Le virus isolé n'avait à l'époque pu être identifié faute de moyens mafériels. de référence aimablement fournis par le docteur JANSEN $(t)$.

\section{Le virus:}

C'est le troisième passage sur cerveau de souriceau nouveau-né d'une souche sauvage d'Afrique du Sud du virus de la maladie de Wesselsbronn et de la Fièvre de la Vallée du Rifł qui a servi de matérial de départ.

\section{L'ontigène :}

L'antigène est obtenu d̀ partir de cerveaux de souriceaux nouveaux-nés morts à la suite de l'inoculation du virus ou parvenus au stade final de la maladie.

30 Les globules rouges:

Les globules rouges d'oic ont été utilisés.

40 Les sérums : $(++)$

Les sérums examinés concernent 273 moutons d'Afrıque Centrale vivant dans des régions géographiques différentes et 33 ruminants sauvages du Tchad.

C'est ainsi qu'ont été analysés :

- 23 sérums de moutons de la région de Mao, au Nord du Tchad,

- 18 sérums de moutons de la région d'Abéché, au Nord Est du Tchad,

- 96 sérums de moutons de la région de Fort-Lamy au Tchad,

- 14 sérums de moutons de la région de Fort-Archambault au Sud du Tchad.

- 63 sérums de moutons de la région de N'Gaoundéré au Nord du Cameroun,

- 59 sérums de moutons de la région de Maroua, à l'extrème Nord du Cameroun,

- 33 sérums d'animaux sauvages du Tchad ainsi répartis :

(+) L'auteur remercie Ici les docteurs JANSEN, WEISS, HOWELL el ERAMUS, WESTHUIZEN et MARE pour leur accueii amical ef pour toutes les possibilités de travail qu'ils ont offert pour érudier les techniques bien partıculières que sont celles utilisées avec les arbovirus comme la peste équine, la Fièvre de la Vallée du Rift, la maladie de Wesselsbronn et la Blue Tongue.

$\left(\frac{1}{+}+\right)$ L'auteur remercie également Messieurs les Vétérinaires Inspecteurs Cheís des secteurs inléressés por cefle enquề e, qui ont bien voulu lui procurer les sérums de moutons demandés. 
a) 25 sérums d'animaux sauvages du Nord et du Nord-Est du Tchad :

- 2 sérums de gazelles rufifrons : Gozella rufifrons (Gray) du Nord-Est du Tchad:

- 12 sérums de gazelles dorcas : Gazella dorcos dorcas (Linné) au Nord-Est du Tchad :

- 1 sérum de gazelle dama : Gazella dama (Pallas) du Nord du Tchad;

- 6 sérums de gazelles dama: Gazella dama (Pallas) du Nord-Est du Tchad ;

- 1 sérum de damalısque : Damaliscus korrigum (Ogilby) du Nord-Est du Tchad ;

- 3 sérums d'oryx : Oryx algazel (Oken) du Nord-Est du Tchad.

b) 8 sérums d'animaux sauvages du Sud du Tchad :

- 2 sérums de gazelle rufifrons: Gazello rufifrons (Gray) ;

- 1 sérum de damalisque : Damaliscus korrigum (Ogilby) ;

- 2 sérums de bubale: Alcelaphus lelwel (Heuglin) ;

- 1 sérum de cob de buffon: Adenota kob (Erxleben) ;

- 1 sérum de cob de roseaux : Redunca redunca (Blaine) :

- 1 sérum de buffle : Syncerus Caffer aequinoxials (Blyth).

\section{B) Techniques.}

La réaction d'inhibution de l'hémagglutination a été utilisée. Les techniques de préparation de l'antigène et de la réaction elle-même étont identıques pour les deux virus, tout ce qui est détaillé ci-dessous concerne indifféremment l'un ou l'autre de ces virus. Le $\mathrm{pH}$ optimum des réactions d'hémagglutination ef d'ınhibition de I'hémagglutination est 6,4 pour la maladie de Wesselsbronn, 6,5 pour la Fièvre de la Vallée du Rift.

\section{L'antigène :}

L'antigène est obtenu d̀ partir de cerveaux de souriceaux nouveaux-nés morts à la suite de l'inoculation du virus de la maladie de Wesselsbronn ou de la Fièvre de la Vallée du Rift, ou, ce qui est préférable, à partir de cerveaux de souriceaux nouveaux-nés parvenus au stade final de la maladie. La préparation de l'antigène fait appel à la méthode de l'extraction à l'acétone ef à l'éther. Cette méthode est exactement celle utilisée et décrite en détail par CLARKE ef CASALS (2) dans leur article fondamental sur la préparation des antigènes hémagglutinants pour l'étude des arbovirus. Les dilutions d'antigène sont faites en tampon boraté à $\mathrm{pH} 9$ à 4 p. 100 d'albumine bovine.

\section{$2^{\circ}$ Les globules rouges.}

On utilise des globules rouges d'oie en suspension à 0,5 p. 100 et prélevés 3 jours avant leur utilisation. Au moment de l'emploi, la dilution à 0,5 p. 100 est préparée dans un tampon acide aux phosphates. Ce tampon contient du chlorure de sodium et des phosphates. II est préparé à partir de deux solutions stocks qui ont la composition suivante :
Solution 1

aâ $\begin{aligned} & (0,15 \mathrm{M} \mathrm{NaCL} \\ & \left(0,2 \mathrm{M} \mathrm{Na}_{2} \mathrm{HPO}_{4}\right.\end{aligned}$
Solution 2

âa $\begin{aligned} & (0,15 \mathrm{M} \mathrm{NaCL} \\ & \left(0,2 \mathrm{M} \mathrm{Na} \mathrm{H}_{2} \mathrm{PO}_{\text {I }}\right.\end{aligned}$
Les proportions de ces deux solutions que I'on doit utiliser pour obtenir le $\mathrm{pH} 6,4$ et 6,5 après mélange à un volume égal de tampon borate à $\mathrm{pH} 9$ sont données $\mathrm{Cl}$-dessous :

\begin{tabular}{|c|c|c|}
\hline $\begin{array}{c}\text { PH final } \\
\text { désiré }\end{array}$ & Solution 1 & Solution 2 \\
\cline { 1 - 2 } 6,2 & 22 & 78 \\
6,4 & 32 & 68 \\
6,6 & 45 & 55 \\
6,8 & 55 & 45 \\
\hline
\end{tabular}

30 Les sérums:

Les sérums de références utilisés sont deux sérums hyperımmuns de mouton, le premier inhibant l'hémagglutınation du virus de Wesselsbronn pour les globules rouges d'oie à la dilution du 1/1024, le second inhibant l'hémagglutination du virus de la Fièvre de la Vallée du Rift à la dilution du 1/1024 également.

Tous les sérums sont traités systématiquement au kaolin lavé aux acides pour éliminer les inhibiteurs non spécifiques et sont adsorbés par des globules rouges d'oie. 
Les 273 sérums précédemment énumérés correspondent à ceux qui ont été examinés. 63 autres sérums de moutons ont été rejetés parce qu'ils présentaient des hémagglutinines non spécifiques, rebelles à toute adsorption au kaolin.

40 Lo réaction d'inhibition de l'hémogglutination :

Le titrage de l'antigène, les réactions qualitatives et quantitatives de l'inhıbıtion de l'hémagglutination sont effectuées sur plaques plexiglass en suivant la technique habituellement employée à Onderstepoort. L'antigène lyophilisé est réhydraté la veille du jour de la réaction. Pour le titrage de celuı-cı la dernière dilution donnant une hémagglutination complète est consıdérée comme l'unité et sert au calcul de la dilution à utiliser dans la réaction d'inhibition de l'hémagglutination. Les antigènes employés hémagglutınaient à la dilution du $1 / 512 \mathrm{e}$. Les réactions qualitatives ef quantitatıves d'inhibition de l'hémagglutınation se font également à $\mathrm{pH}$ 6,4 et 6,5 . On inclut dans ces réactions un témoin globules rouges, un témoin antigène et aussi un témoin sérum pour contrôler l'absence d'hémagglutinines spontanées non adsorbées dans le sérum. Les dilutions sont faites en tampon boraté d̀ pH 9 d̀ 0,4 p. 100 d'albumine. On prend 6 unités antigéniques. L'incubation du mélange sérum $(0,2 \mathrm{ml})$ et antigène $(0,2 \mathrm{ml})$ est d'une nuit à $4^{\circ} \mathrm{C}$ et la lecture est faite après avoir ajouté les globules rouges $(0,4 \mathrm{ml})$ et laissé les plaques à la température ordinaire pendant une heure. On lit en notant la dernière dilution où l'inhibition de l'hémagglutınation est complète.

\section{III. - RÉSULTATS ET DISCUSSION}

\section{A) Résultats.}

706 réactions ont été effectuées : 588 examens qualitatifs et 118 examens quantıtatifs. Les résultats globaux sont rapportés dans le tableau I.

10 Lo moladie de Wesselsbronn :

282 réactions qualitatives et 65 réactions quantıtatives ont été effectuées. L'analyse qualitative a intéressé 251 sérums de moutons et 31 sérums d'animaux sauvages. Les réactions quantitatives ont été effectuées sur 48 sérums positifs de moutons et sur 17 des sérums positifs d'animaux sauvages.

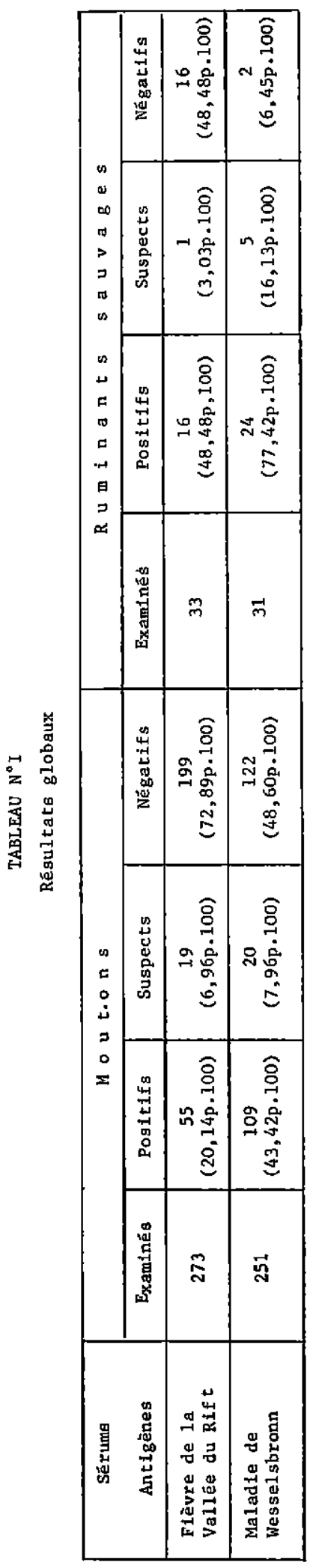


a) Sérums de moutons :

109 sérums sur 251 analysés se sont montrés positifs, soit 43,42 p. 100; 122 se sont montrés négatifs soit 48,60 p. 100:20 ont montré des traces d'anticorps soit 7,96 p. 100 . Le tableau II rend compte de ces résultats.

\section{TABLEAU $N^{\circ} I I$}

Fiềvre de la vallểe du Rift. Maladte de Wesselsbronn

\begin{tabular}{|c|c|c|c|c|}
\hline Pays & Localitë & $\begin{array}{l}\text { Espèce animale } \\
\text { sêrums étudiês }\end{array}$ & $\begin{array}{l}\text { Fièvre de la vallée du Rift } \\
\text { sêrologie positive }\end{array}$ & $\begin{array}{l}\text { Maladie de Wesselsbronn } \\
\text { sêrologie positive }\end{array}$ \\
\hline \multirow{16}{*}{ Tchad } & Mao & Moutons & 2 sur 23 soit $8,69 \mathrm{p} \cdot 100$ & 8 sur 22 soit $36,36 \mathrm{p} .100$ \\
\hline & Moussoro & Gazelle dama & 0 sur 1 & $1 \operatorname{sur} 1$ \\
\hline & \multirow{6}{*}{ Abềché } & Moutons & 1 sur 18 soit $5,55 \mathrm{p} .100$ & 7 sur 15 soit $46,66 \mathrm{p} .100$ \\
\hline & & Gazelle rufifrons & $2 \operatorname{sur} 2$ & 2 sur \\
\hline & & Gazelle dorcas & 4 sur 12 & 8 sur 24 \\
\hline & & Gazelle dama & 2 sur 6 & 5 sur \\
\hline & & Damal isque & 1 sur 1 & $1 \operatorname{sur} 1$ \\
\hline & & Oryx & 2 sur 3 & 2 sur 3 \\
\hline & Fort-Lamy & Moutons & 7 sur 96 solt $7,29 p \cdot 100$ & 32 sur 96 solt $33,33 \mathrm{p} \cdot 100$ \\
\hline & \multirow{7}{*}{$\begin{array}{c}\text { Fort- } \\
\text { Archambault }\end{array}$} & Moutons & 4 sur 14 soit $29,57 \mathrm{p} \cdot 100$ & 8 sur 8 soit 100 p. 100 \\
\hline & & Gazel1e rufifrons & 1 sur 2 & 2 sur 2 \\
\hline & & Damalisque & 0 sur 1 & 1 sur $I$ \\
\hline & & Bubale & 0 sux 2 & 1 sur 2 \\
\hline & & Cob de buffon & 0 sur 1 & \\
\hline & & Cob des roseaix & 1 sur 1 & $0 \operatorname{sur} 1$ \\
\hline & & Buffle & 1 sur 1 & 1 sur 1 \\
\hline \multirow{2}{*}{ Cameroun } & N'Gaoundêrê & Moutons & 14 sur 63 soit $22,22 \mathrm{p} .100$ & 15 sur 56 soit $26,78 p .100$ \\
\hline & Maroua & Moutions & 27 sur 59 soit $45,76 p \cdot 100$ & 39 sur 54 solt $72,22 \mathrm{p} .100$ \\
\hline
\end{tabular}

b) Sérums d'animaux sauvages:

24 sérums d'animaux sauvages sur 31 analysés se sont montrés positifs, solt 77,42 p. 100 ; 2 se sont montrés négatifs solt 6,45 p. 100 et 5 ont montré des traces d'anticorps soit 16,13 p. 100. Le tableau IV rend compte de ces résultats.

20 Fièvre de la Vallée du Rift :

306 réactions qualitatives et 53 réactions quantıtatives ont été effectuées. L'analyse qualitative a intéressé 273 sérums de moutons et 33 sérums d'animaux sauvages. Les réactions quantitatives ont été effectuées sur 41 des sérums positifs de moutons et sur 12 des sérums positifs d'animaux sauvages. a) Sérums de moutons :

55 sérums de moutons sur 273 se sont montrés positifs, soit 20,14 p. 100 ; 199 se sont montrés négatıfs, soit 72,89 p. $100 ; 19$ ont montré des traces d'anticorps, soit $6,96 \mathrm{p} .100$. Les résultats sont rapportés sur le tableau III.

\section{b) Sérums d'animaux sauvages :}

16 sérums d'animaux sauvages sur 33 analysés se sont montrés positifs, soit 48,48 p. 100 ; 16 se sont montrés négatifs, soit 48,48 p. 100 également et un des sérums a montré des traces d'anticorps, solt 3,03 p. 100. Le tableau IV donne le détail de ces résultats. 
TABLEAU III. - Recherche des anticorps contre le virus de la fièvre de la Vallée du Rift chez les moutons

\begin{tabular}{|c|c|c|c|c|c|}
\hline Pays & Localitê & $\begin{array}{l}\text { Sérums } \\
\text { examinés }\end{array}$ & $\begin{array}{l}\text { Sérums } \\
\text { suspects }\end{array}$ & $\begin{array}{l}\text { Sérums } \\
\text { positifs }\end{array}$ & $\begin{array}{c}\text { Taux d'inhibition } \\
\text { observês }\end{array}$ \\
\hline \multirow{4}{*}{ Tchad } & Mao & 23 & 2 & $2(8,69 \mathrm{p}, 100)$ & $(2)^{+} 1 / 40$ ว̀ $1 / 80$ \\
\hline & Abéché & 18 & 1 & $1(5,55 \mathrm{p} .100)$ & (1) $1 / 160$ \\
\hline & Fort-Lamy & 96 & 0 & $7(7,29$ p. 100$)$ & (4) $1 / 20$ à $1 / 160$ \\
\hline & $\begin{array}{l}\text { Fort- } \\
\text { Archambault }\end{array}$ & 14 & 5 & $4(28,57$ p. 100$)$ & (3) $1 / 40$ à $1 / 160$ \\
\hline \multirow{2}{*}{ Cameroun } & N'Gaoundērë & 63 & 4 & $14(22,22, p .100)$ & (12) $1 / 10$ à $1 / 320$ \\
\hline & Maroua & 59 & 7 & $27(45,76 \mathrm{p} .100)$ & (18) $1 / 10$ à $1 / 320$ \\
\hline
\end{tabular}

+ Les chiffres entre parenthèses indqquent le nombre de sêrums examinés quantilativement.

TABLEAU IV. - Examens des sér ums des rumınants sauvages

\begin{tabular}{|c|c|c|}
\hline Espèce animale & $\begin{array}{l}\text { Anticorps contre le virus } \\
\text { de la Fièvre de la Vallée du Rift }\end{array}$ & $\begin{array}{l}\text { Anticorps contre le virus de } \\
\text { la Maladie de Wesselsbronn }\end{array}$ \\
\hline $\begin{array}{c}\text { Gazeita mififrons } \\
1 \\
2 \\
3 \\
4\end{array}$ & $\begin{array}{l}+(1 / 10) \\
+(1 / 40) \\
+(1 / 20) \\
-\end{array}$ & $\begin{array}{l}+(1 / 10) \\
+(1 / 160) \\
+(1 / 40) \\
+\end{array}$ \\
\hline $\begin{array}{c}\text { Gazella doma } \\
1 \\
2 \\
3 \\
4 \\
5 \\
6 \\
7\end{array}$ & $\begin{array}{l}- \\
- \\
+(1 / 80) \\
+(1 / 10) \\
- \\
-\end{array}$ & $\begin{array}{l}+ \\
+(1 / 20) \\
+(1 / 40) \\
+(1 / 40) \\
+(1 / 10) \\
\text { traces } \\
+(1 / 320)\end{array}$ \\
\hline $\begin{array}{c}\text { Gazetza dorcas doreas } \\
1 \\
2 \\
3 \\
4 \\
5 \\
6 \\
7 \\
8 \\
9 \\
10 \\
11 \\
12\end{array}$ & $\begin{array}{l}- \\
- \\
- \\
+(1 / 10) \\
+(1 / 160) \\
= \\
= \\
+ \\
+ \\
+(1 / 160)\end{array}$ & $\begin{array}{l}+(1 / 160) \\
- \\
\text { non examiné } \\
+(1 / 10) \\
+(1 / 80) \\
+ \\
+ \text { traces } \\
+(1 / 20) \\
+ \\
+ \\
+ \\
+\end{array}$ \\
\hline $\begin{array}{cl}\text { Damaliscus } & \text { 2. korrigum } \\
1 \\
2\end{array}$ & $+(1 / 10)$ & $+(1 / 40)$ \\
\hline $\begin{array}{c}\text { Alcephalus letwel } \\
1 \\
2\end{array}$ & - traces & $+\underset{t r a c e s}{(1 / 160)}$ \\
\hline $\begin{array}{c}\text { Oryx aigazel } \\
1 \\
2 \\
3\end{array}$ & $\begin{array}{l}- \\
+(1 / 10) \\
+(1 / 40)\end{array}$ & $\begin{array}{r}\text { traces } \\
+(1 / 10) \\
+(1 / 10)\end{array}$ \\
\hline $\begin{array}{l}\text { Adenota kob } \\
\text { Redunca redunca }\end{array}$ & $\overline{+}$ & $\begin{array}{l}\text { non testê } \\
\text { traces }\end{array}$ \\
\hline Syncemis caffer aequinoxialis & $\leftarrow(1 / 10)$ & $+(1 / 320)$ \\
\hline
\end{tabular}

$+(1 / x)$ : Sêrum positif, Inhibant totalement 1 'hémagglutination à la dilution de $1 / x$.

+ : Sérum positif à l'analyge qualitative (étude quantitative non falte).

- : Sêrum négatif. 
30 Répartition de ces deux arboviroses por régıon.

Le tableau $V$ donne une idée de l'incidence respective de chacune de ces viroses dans les différentes régions prospectées. La carte ci-jointe permef de situer les localités correspondantes.

TABLEAU V. - Répartition régionale des deux arbovivores

\begin{tabular}{|c|c|c|c|c|}
\hline Pays & Localité & Espèce animale & $\begin{array}{l}\text { Fiève de la vallêe du Rift } \\
\text { Sêrologie positive }\end{array}$ & $\begin{array}{l}\text { Maladie de Wesselsbronn } \\
\text { Sérologle positive }\end{array}$ \\
\hline \multirow{16}{*}{ Tchad } & Mao & Ouis axies & $2(23)^{+} 8,69 \mathrm{p} .100$ & 8 (22) 36,36 p. 100 \\
\hline & Moussoro & Gazelta dama & $0 \quad(1)$ & 1 (1) \\
\hline & \multirow{6}{*}{ Abêché } & ovis aries & 1 (I8) 5,55 p. 100 & 7 (15) 46,66 p. 100 \\
\hline & & Gazeliza rufifrons & $2(2)$ & 2 (2) \\
\hline & & Gazelta doreas dorcas & $4(12)$ & $8(11)$ \\
\hline & & Gazelía dama & $2(6)$ & $5(6)$ \\
\hline & & Daraliscus 2.korrioum & 1 (1) & $1(1)$ \\
\hline & & Ormix algazel & $2(3)$ & 2 (3) \\
\hline & Tort-Lamy & ovis aries & 7 (96) 7,29 p.100 & 32 (96) 33,33 p. 100 \\
\hline & \multirow{7}{*}{$\begin{array}{c}\text { Fort- } \\
\text { Archambau1t }\end{array}$} & ovis aries & $4(14) 28,57$ p. 100 & 8 (8) $100 \mathrm{p} \cdot 100$ \\
\hline & & Gazelza mufifrons & $1 \quad(2)$ & $2 \quad(2)$ \\
\hline & & Domaliscus t.korrigum & $0 \quad(1)$ & 1 (1) \\
\hline & & Ateephatus tetwel & $0 \quad(2)$ & $I\langle 2\rangle$ \\
\hline & & Adenota kob & $0 \quad(1)$ & \\
\hline & & Redunca redunca & 1 (1) & $0 \quad(1)$ \\
\hline & & $\begin{array}{l}\text { Syncems cafjer } \\
\text { aequinoxialis }\end{array}$ & $1 \quad(1)$ & 1 (1) \\
\hline \multirow{2}{*}{ Cameroun } & N'Gaoundêrê & Ovis ames & 14 (63) 22,22 p.100 & 15 (56) $26,78 \mathrm{p} .100$ \\
\hline & Maroua & ovis aries & $27(59) 45,76 \mathrm{p} .100$ & 39 (54) $72,22 \mathrm{p} .100$ \\
\hline
\end{tabular}

+ Le prenier chiffre donne le nombre de sérums positifs, le second, entre parenthêses, celui des sérums examinés.

\section{B) Commentaires.}

10 Un pourcentage relativement élevé de moutons possède des antıcorps contre la Fièvre de la Vallée du Rift et la maladie de Wesselsbronn. Le degré d'endémicité de cette dernière est plus élevé que celui de la première affection cltée et ceci dans toutes les régions.

20 Les ruminants sauvages sont le témoin d'une infection par ces deux virus dont la trace est révélée par les anticorps spécifiques présents dans leur sérum. Cette constatation mérite d'être soulignée. En effet, le rôle de ces vertébrés dans l'histoire naturelle des zoonoses a été d'une façon générale peu étudié. On s'est beau- coup préoccupé des arthropodes vecteurs aux dépens du réservair naturel d'infection.

30 Toutes les régions qui ont été intéressées par cette enquête connaissent le virus de Wesselsbronn et celui de la Fièvre de la Vallée du Rift. Il semblerait cependant que ce dernier soit moins répandu à Fort-Lamy et dans les régions sahéliennes situées au Nord de cette ville.

40 II a été constaté depuis quelques années un grand nombre d'avortements chez les bovins de la région de Maroua au Cameroun. Le problème de leur éfiologie n'a pas été élucidé de façon définitive.

En effet, dans la symptomatologie propre d̀ chaque type d'avortement on retrouve toujours à peu près les mêmes descriptions et il est rap- 
tiquement impossible de fonder un diagnostic uniquement sur des symptômes et des lésions. Les enquêtes sérologiques sur la brucellose, la Fièvre Q, les néorickettsioses n'ont pas permıs d'expliquer la majorité des avortements. De nombreuses hypothèses ont été envisagées : maladies chroniques (brucellose) rickettsioses (Fièvre Q), néorickettsioses, affections à gros virus du type lymphogranulomatose, viroses diverses qui dans leur phase aiguë peuvent provoquer l'avortement, maladies parasıtares comme la gale, la strongylose qui épuisent les femelles gestantes, carences simples ou multiples en minéraux ou vitamınes, intoxications par certaines plantes, etc... Une pathologie variée intervient, et, quand on constate au terme de cette enquête que 45,76 p. 100 des sérums de moutons de la région de Maroua présentent des anticorps contre la Fièvre de la Vallée du Rift, il est permis de se demander si cette virose ne vient pas s'ajouter à l'étiologie déjà complexe des avortements des bovins dans ces régions.

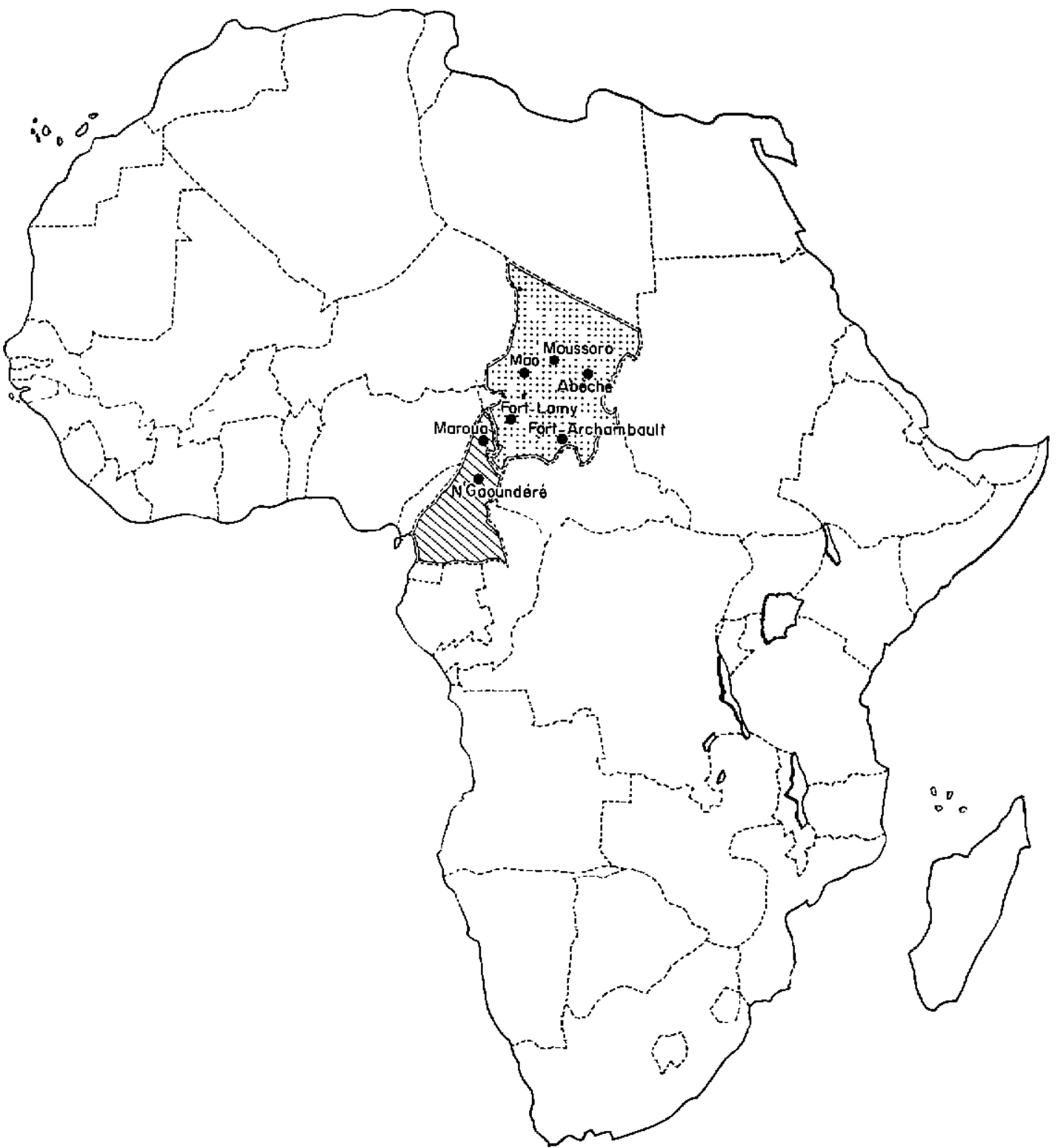

Répartıtion régionale de la maladie de Wesselsbronn et de la fièvre de la vallée du Rift. 


\section{CONCLUSION}

La Fièvre de la Valiée du Rift ef la maladie de Wesselsbronn existent à l'état enzootique en Afrique Centrale chez les moutons et les anımaux sauvages et pourraient expliquer certains avortements d'origine inconnue.

On n'a que rarement prêté attention à ces deux affections qui jouent vraisemblablement un rôle dans la pathologie des petits ruminants ef peut-être des bovins. Aussi a-t-il paru utile de rappeler les aspects cliniques et nécropsiques de ces deux affections.

\section{Institut d'Elevage ef de Médecine vétérnaire des Pays tropicaux. \\ Laboratoire de recherches vétérinoires de Farcho, Fort-Lamy, Tchad.}

N. B. : Ce texte était rédigé quand nous avons eu connaissance de l'article «Immunologie des Arbovirus chez les Pygmées Babinga de Centrafrique $\gg$, de A. CHIPPAUX et Cl. CHIPPAUXHYPPOLYTE de l'Institut Pasteur de Bangui, paru dans le dernier numéro du Bulletin de la Société de pathologie exotique (1965, no 5 , p. 820). Les auteurs ont montré que 2,8 p. 100 , 7 p. $100,12,5$ p. 100 et 26 p. 100 des sérums humains de différentes rribus de Centrafrique présentaient des anticorps inhibant l'hémagglutination vis-à-vis du virus de Wesselsbronn ce qui confirme également l'existence et la diffusion de ce virus en Afrique Centrale.

\section{SUMMARY}

First serological record on the incidence of Wesselsbronn's Disease and Rift Valley Feyer in sheeps and wild ruminants in Chad ans Cameroun

Existence of antibodies against Wesselsbronn's and Rift Valley Fever virus, often at a high titre, has been shown by use of Hemagglutination Inhibition test, in small ruminants in Ched and Cameron and in wild ruminants in Chad. In some areas of Cameron, $72 \mathrm{p}, 1 \mathrm{CO}$ of the sheeps have been shown to give a posilive reaction to Wesselsbronn's virus, and 45 p. 100 to be positive to Rift Valley Fever virus. Though both diseases had been suspected by some authors, they were never recorded, reported or described in these countries. This is not very surprising, in view of the very discrete symptoms of Wesselsbronn Disease which are often unnoticeable and the existence, in some African regions of unapparent cases of Rift Valley Fever, which could explain the etiology of some disconcerting abortions in cattle.

Gazelle, topi, hartebeest, oryx, reedbuck and buffalo have probably a role in the epizootiology of this infection.

\section{RESUMEN}

Primeras notas serológicas sobre la incıdencia de la enfermedad de Wesselsbronn y de la fiebre del valle del Rift en las ovejas y los rumiantes salvajes de Chad y de Camerún

Utilizando la lécnica clásica de inhibición de la hemaglutinación, el autor demostró la presencia de anticuerpos contra la enfermedad de Wesselsbronn y la fiebre del valle del Rifi, con una tasa frecuentemente elevada, en los pequeños rumiantes de Chad y de Camerún y en los rumiantes salvajes de Chad.

En algunas regiones de Camerún, se encontraron, en 72 p. 100 de ovejas, anticuerpos indicando la presencia de la enfermedad de Wesselsbronn, $y$ en 45 p. 100 de el las anticuerpos contra la enfermedad del valle del Rift.

Estas dos enfermedades fueron sospechadas pero no fueron nunca reconocidas o descritas en los dos paises. Eso no es sorprendente cuando se conoce la sintomatologia muy incompleta de la enfermedad de Wesselsbronn, frecuentemente no descubierta, y la existencia, en algunas regiones de Africa, de formas 


\begin{abstract}
inaparentes de la Fiebre del Valle del Rıft. La existencı de la última podria explicar ciertos abortos cuya etiologıa quedaba desconocida hasta ahora en los bovinos.

La gacela, el damaliscus, el búbalo, el Oryx. el cob, el búfalo verosimilmente desempeñan un papel en la epizootiologia de esta enfermedad.
\end{abstract}

\title{
BIBLIOGRAPHIE
}

1. ALEXANDER (R. A.), DICKSON (J.). La Fièvre de la Vallée du Rift dans I'Union. J. S. ofr. vet. med. Ass., 1951, 22, (3) 105-112.

2. CLARKE (D. H.) et CASALS (J.). - Techniques pour l'hémagglutination ef l'inhibition de l'hémagglutination avec les arbovirus. Am. J. Trop. Med. 1958, 7, 561.

3. DAUBNEY (R.), HUDSON (J. R.). - La Fièvre de la Vallée du Rift. Lancet 1932, 222, 611-612.

4. DAUBNEY (R.), HUDSON (J. R.). - La Fièvre de la Vallée du Rift. East African med. Journal, 1933, 10, (1), 2-19 (cité par Kaschula 1953 et Henning 1956).

5. DAUBNEY (R.), HUDSON (J, R.), GARNHAM (P. C.). - Une maladie non décrite du mouton, du bétail et de l'homme en Afrique de l'Est. Hépatite enzootique ou Fièvre de la Vallée du Riff. J. Path. Bact. 1931, 34, 545-579.

6. FERGUSON (W.). - Identification de la Fièvre de la Vallée du Rift en Nigéria. Bull. Epiz. Dis. Afr. 1959, 7, 319.

7. FINDLEY (G. M.), DAUBNEY (R.). Le virus de la Fièvre de la Vallée du Rift ou de l'hépatite enzootique. Lancet, 1931, 221, 1350-1351.

8. FINDLAY (G. M.), STEFANOPOULO (G. M.), MACCALUM (F. O.). - Présence d'anticorps contre la Fièvre de la Vallée du Rift dans le sang des africains. Bull. Soc. Path. Exot. 1936, 29, 986-994 (cité par Kaschula, 1953).

9. GEAR (J.), DE MEILLON (B.), MEASROCH (V.), HARWIN (R.), DAVIS (D. H.S.). La Fièvre de la Vallée du Riff en Afrique du Sud. L'apparition de cas humains de la maladie dans l'état libre d'Orange, le nord-ouest de la province du Cap, l'ouest et le sud du Transvaal. Recherches sur le terrain ef au laboratoire. S. afr. Med. J.1951. 25, 908-912.

10. MUNDEL (B.), GEAR (J.). - La Fièvre de la Vallée du Rift. L'existence de cas humains à Johannesbourg. S. ofr. Med. J. 1951, 25, 797-800.

11. PELISSIER (A.) et ROUSSELOT (R.). Enquête sérologique sur l'incidence des virus neurotropes chez quelques singes de l'afrique équatoriale française. Bull. Soc. Path. Exot. 1963, 56, 4, p. 586.

12. SCHULZ (K.). - La pathologie de la Fièvre de la Vallée du Rifl ou de l'hépatite enzootique en Afrique du sud. J. 5. ofr. vet. med. Ass. 1951, 22 (3), 113-120.

13. SCHULZ (K. H.). - La Fièvre de la Vallée du Rift en Afrique du sud. Rapport spécial $n^{\circ}$ 5/51. Union Dept. of Health-Plague Res. Lab., 1951, p. 1-26 (cıté par Kaschula 1953).

14. SCOTT (G. R.), WEDDEL (W.), DAPHNE REID. - Premières constatations sur la fréquence de la Vallée du Rift chez les bovins au Kenya. Bull. Epiz. Dis. Afr. 1956, 4. p. 97-101.

15. SMITHBURN (K. C.), HADDOW (A. J.), GILLETT (J. D.). - La Fièvre de la Vallée du Rift. Isolement du virus à partir de moustiques sauvages, 1948, 29, 107-121.

16. STORDY (R. J.). - Mortalité chez les agneaux. Ann. Rep. Dept. Agrc. Brit. Eost. Africa. 1912-1913. p. 13 (cité par Henning, 1956).

17. WEISS (K. E.), HAIG (D. A.), ALEXANDER (R. A.). - Le virus de Wesselbronn. Un virus nouvellement décrit associé à l'avortement des animaux domestiques. Onderstepoort J. 1956, 27. (2), 183-195. 